Danny Meadows-Klue

is chairman and CEO of the $I A B U K$ and as IAB Europe president was a partner in the Active-Ad project.
Keywords: advertising, future, channels, Web, iTV, wireless, agencies, trends, research, Europe, mobile, communications
Danny Meadows-Klue Interactive Advertising Bureau PO Box 26726

London SW2 4AN UK

Tel: +44 (0)2086839557

E-mail: danny@iabuk.net

\section{Practitioner attitudes to interactive advertising}

\author{
Danny Meadows-Klue \\ Received (in revised form): 5 August 2002
}

\begin{abstract}
The Active-Ad project, a pan-European study co-funded by the European Commission, was set up to analyse the success criteria for interactive advertising as part of the socioeconomic dimension of the Information Society Technologies (IST) Programme. The portion of the project recorded here took the pulse of the digital media industry across Europe. Its aims were to explore the nature of interactive marketing and the further development of models within the industry and the sector itself. Led by a consortium backed by the IAB and other stakeholders, and coordinated by the IEPRC, it represented a major development in understanding within the sector. This paper is a digest of its results. ${ }^{1}$
\end{abstract}

\section{Introduction to the research}

The European Commission-funded pan-European research project called Active Ad explored the models and trends in interactive advertising. The project ran for 18 months and was coordinated on behalf of seven partners by the International Electronic Publishing Research Centre (IEPRC). In addition to the Interactive Advertising Bureau (Europe), the partners included IMD/Pixelpark, Pro-Active International, the London Business School, the Turku School of Economics, the European Association of Communications Agencies and the World Federation of Advertisers. This portion of the research was conducted by the Turku School in Finland. Questionnaires were sent to 392 advertisers and 900 advertising agencies in England, France, Germany, Italy and Spain. In-depth interviews with 40 advertisers and developers of interactive solutions took place. ${ }^{2}$

The research analysed the perceptions of marketers towards advertising through interactive platforms: the Internet, wireless and interactive television. It is intended for directors responsible for their companies' approach to interactive marketing and the structures used to achieve this. The research set out to determine a common framework for understanding interactive advertising and its manifestations, different uses and possibilities. Results of the research show that interactive online advertising is really online marketing, not simply advertising in its traditional offline sense. The research reinforces the validity of interactive media and highlights a range of barriers and opportunities facing the industry. It pinpoints future trends and key issues as well as opportunities for the growth of digital media. Some of the barriers identified, such as clear measurement metrics, ${ }^{3}$ are process issues already being addressed by the IAB and other elements of the industry, while others, such as the 


\section{Interactive online advertising is really online marketing, not simply advertising in its traditional sense}

penetration of interactive television, are technology adoption issues that appear to be changing more gradually. Among organisational changes uncovered was a failing in the structure of marketing departments and agencies that is seen as restricting the progression of digital advertising and actually hobbling interactive marketing.

\section{Themes}

In the more mature European markets like the UK and Finland, Internet advertising already accounts for more than 1 per cent of total advertising spend $^{4}$ and continues to grow, in general faster than any other medium. It also accounts for a much greater share of its audience's media consumption. Interactive marketing should be integrated into wider marketing communications and be treated as a complementary channel.

According to the research's co-author Robert Picard, a professor at the Turku School of Economics, 'The most important thing that advertisers need to understand is that interactive media are complementary to advertising campaigns in other media, not a replacement. Most types of interactive campaigns work best when involving some sort of cross-media campaign and when involving more than one type of new media.' 5

The research reinforces the validity of these new media, explores marketers' perceptions of them and clarifies the barriers and opportunities. However, it also uncovers a failing in the structure of marketing departments and agencies that sometimes restricts take-up and progression of opportunities in digital marketing due to inherent flaws in their structure. There remains a need for marketers and their agencies to adapt their process and structures to embrace interactivity more effectively.

Overwhelmingly the research identifies that interactive online advertising is really online marketing in its broadest sense, not simply advertising in its traditional model. According to Picard, 'It is a misnomer to refer to interactive campaigns as interactive advertising because their strongest effectiveness comes in other marketing activities such as sales promotion and direct selling. ${ }^{6}$ Interactive marketing is perceived by practitioners as being most effective when combined with other advertising and marketing activities, when it can become very effective for building brand awareness and preference. It creates mechanisms for personal communications that are more effective than those marketers have had access to in the past.

The falling costs of Internet-based communication and the removal of barriers to entry for a greater number of advertisers will see the medium continue to experience growth - albeit at a slower rate than the recordbreaking growth experienced in the late 1990s. Away from the Web, the costs of mobile communication will keep small advertisers out for some time; similarly the costs for interactive television will keep it a large advertiser activity for some time.

According to the team at the IAB, the research suggests that interactivity is often misunderstood throughout the advertising value chain. While it requires specialist expertise, interactivity can no longer be considered in isolation. The challenge to marketers and their agencies is to integrate 


\section{The Internet is set to become an established marketing tool that is especially oriented to CRM and service marketing}

interactive marketing throughout all of the marketing communications disciplines.

\section{Trends}

Despite being relatively young as an advertising medium, the Internet already enjoys a certain level of maturity. Most major advertisers feel they have significantly explored it - even if only in the form of a corporate website. Any scepticism can generally be attributed to unrealistic expectations, poor understanding and the failure to adopt industry-wide standards - including those developed by the IAB - to support accountability and measurability.

Advertising online across Europe will grow despite current economic uncertainty, although this growth will be more measured as the market begins to mature. Many factors drive this growth, including the continued rapid increase in audience numbers, ${ }^{7}$ a phenomenon unique to interactive media. There is also evidence of small to medium-sized advertisers taking more readily to the Internet.

For direct marketers the study endorsed the Internet as becoming an established marketing tool especially oriented to CRM and service marketing. This will be a major growth area, largely due to its ability to market at a one-to-one level and generate real, meaningful dialogue with consumers.

The Web has already proved itself as both a branding and a direct medium. ${ }^{8}$ A key part of the future of Internet advertising lies in its ability to build brands as a cross-platform campaign medium. In spite of the wealth of brand effectiveness research, including dozens of studies by IAB organisations across Europe, many marketers remain trapped in direct response thinking when it comes to how they develop their Internet strategies. However, this research also shows that direct marketing is leading to an increased focus on payment for results through the measurement of clear ROI metrics, including improved measurability, more reliable and accountable data, more creativity and linkage of online advertising to direct selling online.

\section{Benefits}

The study identified the most important benefits of interactive advertising as being direct communication, its potential for tracking, an effective

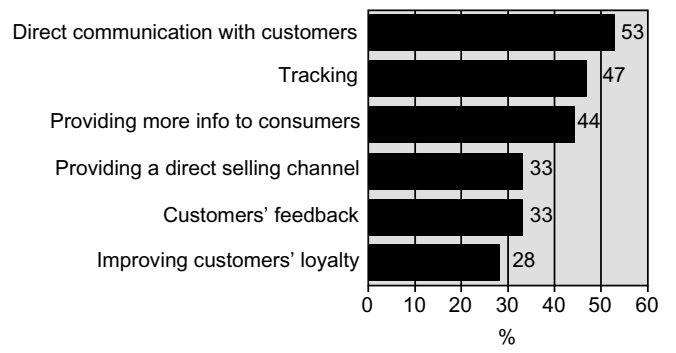

Figure 1: Perception of the benefits of interactive advertising, advertisers' survey. 


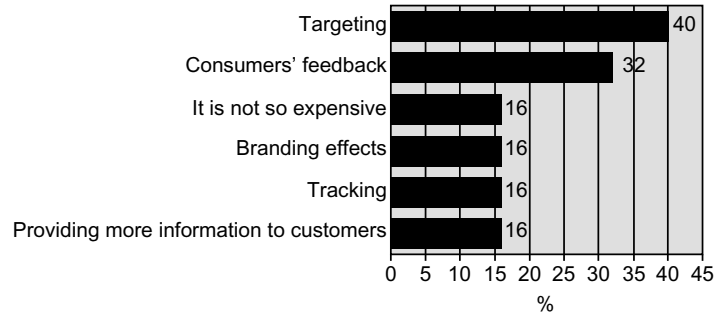

Figure 2: Percentage benefits of interactive advertising from interview results

channel to provide more information to consumers and being a direct selling channel (see Figures 1 and 2).

When quizzed about formats, e-mail was acknowledged as being the most successful thanks to its cost effectiveness, rich layout possibilities, personalisation and short response times, although there was a sense that many marketers did not use brand effectiveness metrics to judge their online campaigns and were using direct response metrics all the time. Among other formats, sponsorships, co-branding and partnerships were cited as creative ways to 'find where consumers are'. Games, quizzes and contests were found to be engaging for users and able to serve many different purposes thanks to their precise targeting. Pop-ups, interstitials ${ }^{9}$ and other forms of rich media were found to be effective in brand building as part of cross-media campaigns.

\section{Wireless}

The research examined mobile devices such as personal digital assistants (PDAs), mobile phones and hand-held computers. It defined the wireless market in Europe in 2001, with 64 per cent of EU citizens possessing a mobile phone - some 235 million people. The EU combined telecom services market is now worth more than $£ 200 \mathrm{bn}$, with an annual growth rate of 12.5 per cent.

While still in an experimental stage, the wireless advertising market is developing. Its attractiveness lies in its mass-market penetration of the access devices. New figures show that the platforms and formats most

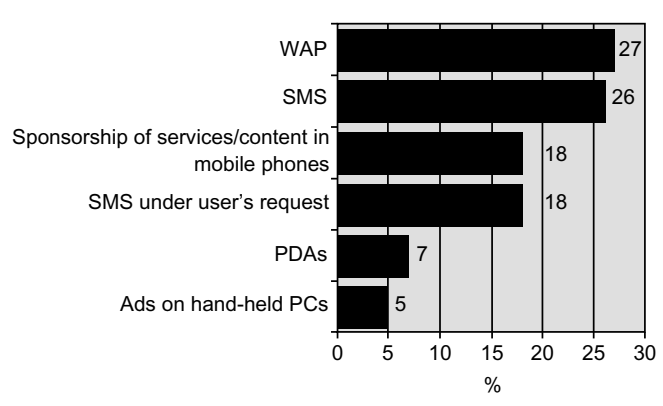

Figure 3: Platforms and formats most used in wireless advertising, advertisers' survey 


\section{The future of mobile advertising depends on improving location systems and improved bandwidth}

\section{The excitement about iTV advertising lies in the expectation for it to deliver one-to-one communications from a mass communication tool}

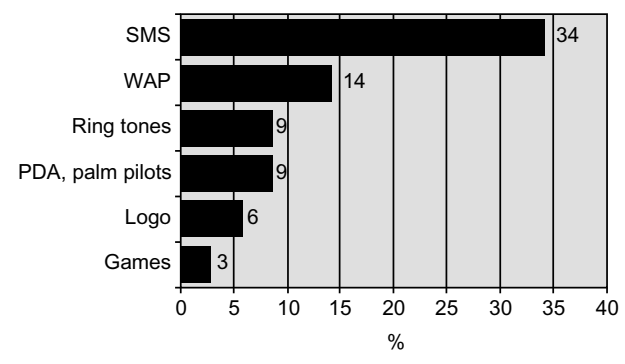

Figure 4: Percentage used in wireless, interviews

used for wireless advertising are WAP, SMS and sponsorship of services/ content in mobile phones (see Figures 3 and 4). The results of the interviews showed that in terms of actual usage, SMS had more than double the usage of WAP, and more than three times that of ringtones.

However, the wireless market faces some specific challenges. According to those questioned, the future of mobile advertising depends on improving location systems that develop new possibilities for advertising as users approach sales points, ensuring adverts become an added-value experience, and improved bandwidth that allows enhanced experiences and faster data downloads. The journey to wireless advertising success also hinges on a number of other critical factors, including managing unsolicited e-mail (spam) and data protection policies. The IAB is also keen to work directly with wireless companies to explore the models for wireless advertising in more detail and develop mapping projects for their relationship to the more established interactive media.

If this can be achieved then those surveyed confirmed that investment in wireless advertising would be considered, but only if wireless proves to improve targeting, increase online purchase rates, improve audience research, improve branding and improve click rates to corporate sites.

\section{iTV}

The research showed that the interactive TV market is still perceived as being years away from being an effective advertising medium. Currently it is limited to a group of big advertisers, in part due to large entrance costs and heavy contractual demands from iTV operators. It is still viewed by advertisers across Europe as an exploratory medium that warrants an experimental approach, and eyes are fixed firmly on the UK where the technology and penetration are more mature.

The excitement about iTV advertising lies in the expectation for it to deliver one-to-one communications from a mass communication tool. It promises perfect targeting for interested potential consumers, with more accurate measurement methods and payment on results. In its favour are accountability and the ability to track consumer behaviour and collect data, and overall it is perceived as safer for commerce than the Internet. Holding back iTV advertising growth are an inability to reach a critical mass that will justify the investment, large production costs, a 


\author{
A greater emphasis \\ on coordination and \\ communication is \\ required if \\ advertisers \\ themselves, and the \\ consultants who \\ service them, are to \\ see the benefits of \\ interactive \\ campaigns
}

requirement to improve metrics further and gaining access to consumer data.

ITV advertising is at the earliest stage of development, although European companies are leading this sector. It has been limited to interactive broadcast advertising in which a viewer can click, for example, on an ordering page; or on advertising or sponsoring electronic programme guides (EPGs), and microsites or walled garden services. These models too are evolving, and in the last 18 months there has been rapid development of the formats and creativity employed.

\section{Failing structures}

The research went further than taking the pulse of Europe's digital market today and examining the trends for the future. It also examined the structure of the industry to identify barriers to growth. This uncovered areas, such as education and perceptions of how the sector worked, but fundamentally pointed to a failing in the structure of marketing departments and agencies, which were seen as restricting take-up and progression of opportunities in digital advertising and actually hobbling interactivity.

Dr Brian Blunden, chairman of the IEPRC - the media network that coordinated the whole Active Ad project - feels that it is a difficult challenge and that internal structures are holding interactivity back. 'It's a tall order, but marketers and their agencies need to come together more effectively and create a network business that will deliver the solution. Both marketing departments and agencies are structured incorrectly to meet these needs. That must change. ${ }^{10}$

The study found that the majority of marketers place interactivity within advertising departments or agencies. This negates or downgrades the importance of sales promotion, direct and PR channels. For this reason marketers are not getting the most out of interactivity. According to Picard, 'Interactive activities are far too useful to be left to the advertising manager alone. Companies need to ensure that teams of from marketing, sales and customer service are involved. ${ }^{11}$ He believes that the other marketing elements make interactive more powerful and that is why the medium is missing many opportunities.

The reason for the apparently detrimental focus through advertising is the dominant structures that prevail in marketing. Essentially responsibilities are too linear, with advertisers focused on generating activity via specialist agencies or via internal departments that operate in partial isolation. In addition to problems with free-flowing communication, the more complex and linear the structures the slower the decision-making process. Picard says that 'interactivity is not the responsibility of one specialist and decisions need to happen fast'. ${ }^{12}$

Although many agencies have consolidated by folding interactive arms into the body of their parent business, this has generally been driven by cost cutting rather than a recognition that the structure is hindering interactivity or that integrating digital specialists with other channel specialists will improve interactivity. Simply bringing a new media team in-house does not tackle the problem, as the structural relationships 
There needs to be a wider consideration for integrating the whole marketing process between digital media and other channels can be managed successfully even if the agency is a stand-alone unit or separate organisation. A greater emphasis on coordination and communication is required if advertisers themselves, and the consultants who service them, are to see the benefits of interactive campaigns. 'There is no one-fits-all solution,' says Picard. 'What's right for Procter \& Gamble is not right for British Airways. What it boils down to is teams of marketers across all disciplines saying together "what can we do to aid interactivity?". There's too much conflict and too many agendas. Marketing should become more coordinated into work teams and structures that facilitate interactivity. ${ }^{, 13}$

The accusation that media specialisation hampers interactivity is not new, but with so many digital media arriving so quickly, this may prove to be the necessary catalyst to a long-awaited restructuring and advertisers as much as agencies need to wake up to this need for change. Picard believes that the 'slow speed of traditional advertising and marketing planning and implementation cycles won't work in interactive advertising because you must be able to react rapidly to customer-initiated interaction or lose the benefits of interactivity'. ${ }^{14}$

Picard also cites the necessary changes to companies' operational structures and activities being underpinned by integrated marketing communications. The research concludes that there needs to be a wider consideration for integrating the whole marketing process, making it a single stream for users that will provide consistent, uniform and coherent messages to consumers. 'Interactivity allows dialogue which leads to data capture for later analysis and use. Adopting such thinking will lead from mass marketing to mass customisation,' says Picard. ${ }^{15}$

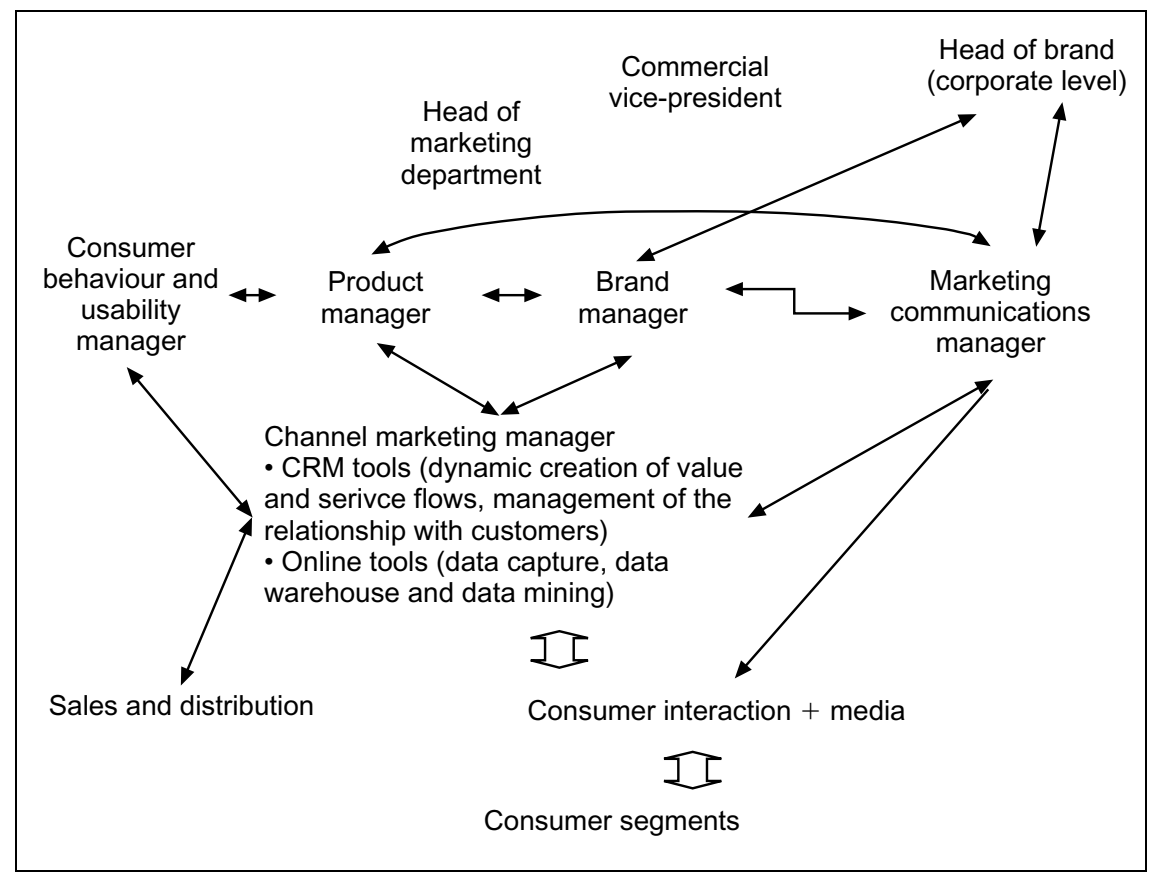

Figure 5: Proposed marketing activity organisation for effective interactive media use 


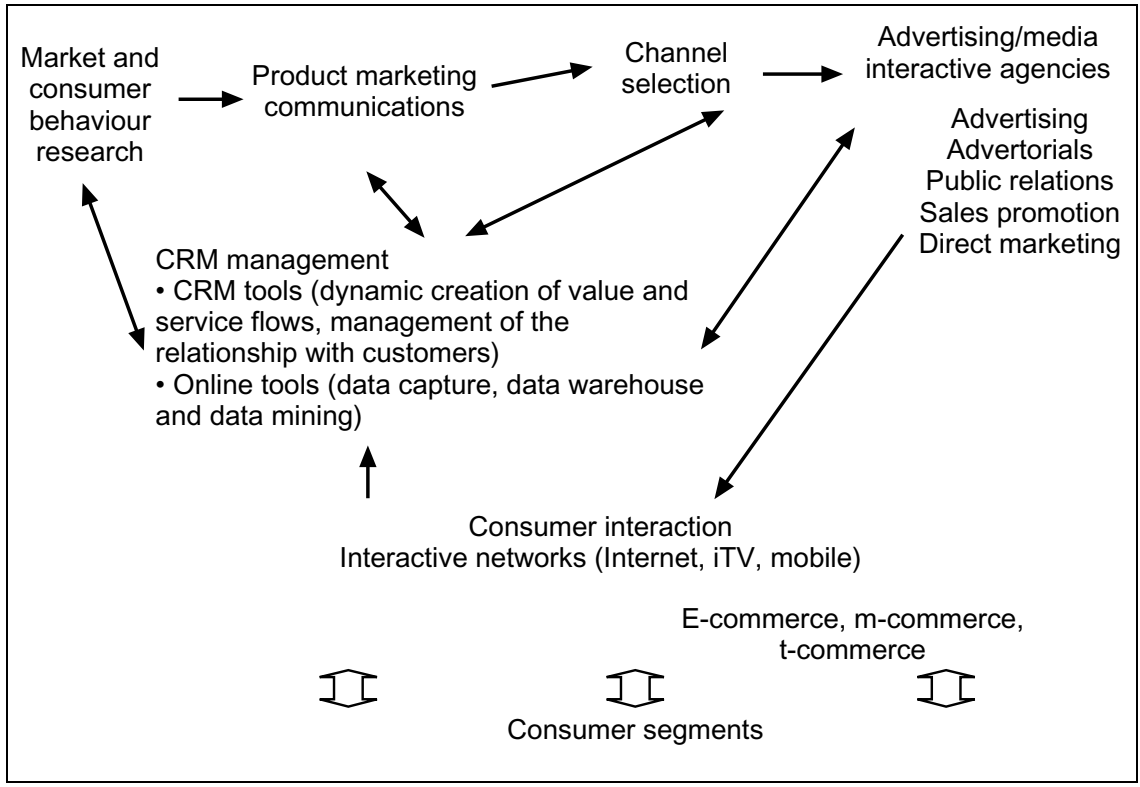

Figure 6: Proposed marketing value chain for the interactive environment

\section{Summary}

Interactive media are a rapidly growing part of the marketer's toolbox and are enjoying long-term rapid audience growth, but spend levels lag well behind audience levels. Marketers are still cautious about interactive media. They know they offer opportunities for marketing communications that go beyond advertising, but they are uncertain on how to use them in the digital marketing mix and are unclear about the benefits from different formats.

Across Europe, advertisers recognise the Internet as one element in a cross-media advertising strategy, and that e-mail, sponsorship and games can be very effective in getting brands closer to consumers. Advertisers increasingly prefer the interactive 'rich media' to static artwork formats.

When it comes to wireless, there is limited knowledge of the possibilities it offers. Currently GSM networks and SMS offer the best opportunities, but critical mass is still an issue, as is dealing with spam, and the advertising model itself remains in its infancy. Until iTV reaches critical mass and costs fall, only big advertisers will continue to test opportunities outside walled gardens, EPG, content and interactive broadcast ads.

A major barrier restricting growth across the Internet is the improvement of metrics and creativity, although part of the industry's challenge is simply spreading best practice. In the wireless sector, location and time-related ads will increase impulse buying and lead to great acceptance of mobile commerce, with the next generation of GPRS and UMTS technology pointing to richer possibilities for creativity and relevancy. ITV has a long way to go; first it needs a critical mass that straddles market acceptance and development.

Picard believes that marketing departments and strategies are too 
Interactivity is about immediacy

\section{Digital media across Europe are in good shape, but without some fundamental infrastructure changes they will not reach their potential}

compartmentalised and insular. Interactivity is about immediacy - which is not served well by current structures. Interactivity is also equally aligned to direct marketing, selling and customer service. When this mind-set is broken, the speed at which marketers can deliver on their interactive objectives will increase and the digital medium will have more opportunity to flourish alongside established media.

All in all, digital media across Europe are in good shape, but without some fundamental infrastructure changes they will not reach their potential. The Active-Ad research programme, thanks to the work of the partners, the IEPRC and the support of the European Commission, has allowed understanding of these media to move forward and the papers are worthwhile reading for the directors of all companies operating in this space.

\section{References}

1. International Electronic Publishing Research Centre (2002) Active-Ad Research: Full Report, IEPRC. Copies of the full research reports can be purchased from the IEPRC (admin@ieprc.org). A seminar slide show of the results of this element of the work developed by the Turku School of Economics, along with research papers in related areas such as advertising expenditure trends and advertising effectiveness studies, are available from the IAB.

2. Although the study was run across several major European markets, individual national findings were not analysed as many of those surveyed had pan-European responsibilities. However, the general themes seem to be common across all markets, although the timing of them may vary depending on the market's maturity.

3. IAB (2002) 'The guide to Internet Advertising Standards', IAB.

4. IAB (2002) 'Internet media spend', IAB.

5. IEPRC, ref. 1 above.

6. Ibid.

7. IAB (2002) 'Growing reach of Europe's Internet audiences', IAB.

8. Milward Brown/IAB (1997); IAB/Dynamic Logic (2000); Taylor Nelson Sofres/IAB (2002).

9. IAB (2002) 'The guide to creative formats for Internet advertising', IAB.

10. IEPRC, ref. 1 above.

11. Ibid.

12. Ibid.

13. Ibid.

14. Ibid.

15. Ibid. 